\title{
Article \\ Layer-by-Layer Electrode Fabrication for Improved Performance of Porous Polyimide-Based Supercapacitors
}

\author{
Niranjala Fernando ${ }^{1,+}$, Hugo Veldhuizen ${ }^{2,+}{ }^{+}$, Atsushi Nagai ${ }^{3}$, Sybrand van der Zwaag ${ }^{2}$ \\ and Amor Abdelkader $1, *$ (D)
}

1 Department of Engineering, Talbot Campus, Bournemouth University, Fern Barrow, Poole BH12 5BB, UK ; nweerahannadige@bournemouth.ac.uk

2 Faculty of Aerospace Engineering, Delft University of Technology, Kluyverweg 1, 2629 Delft, The Netherlands; h.v.veldhuizen@tudelft.nl (H.V.); s.vanderzwaag-1@tudelft.nl (S.v.d.Z.)

3 Department of Electrical and Electronic Information Engineering, Toyohashi University of Technology, Hibarigaoka-1-1 Tenpakucho, Toyohashi 441-8580, Japan; nagai.atsushi.rn@tut.jp

* Correspondence: aabdelkader@bournemouth.ac.uk

+ These authors contributed equally to this work.

Citation: Fernando, N.; Veldhuizen, H.; Nagai, A.; van der Zwaag, S.; Abdelkader, A. Layer-by-Layer Electrode Fabrication for Improved Performance of Porous PolyimideBased Supercapacitors. Materials 2022, 15, 4. https://doi.org/ $10.3390 / \mathrm{ma} 15010004$

Academic Editor: Alina Pruna

Received: 1 December 2021

Accepted: 16 December 2021

Published: 21 December 2021

Publisher's Note: MDPI stays neutral with regard to jurisdictional claims in published maps and institutional affiliations.

Copyright: (C) 2021 by the authors. Licensee MDPI, Basel, Switzerland. This article is an open access article distributed under the terms and conditions of the Creative Commons Attribution (CC BY) license (https:// creativecommons.org/licenses/by/ $4.0 /)$.

\begin{abstract}
Nanoporous polymers are becoming increasingly interesting materials for electrochemical applications, as their large surface areas with redox-active sites allow efficient adsorption and diffusion of ions. However, their limited electrical conductivity remains a major obstacle in practical applications. The conventional approach that alleviates this problem is the hybridisation of the polymer with carbon-based additives, but this directly prevents the utilisation of the maximum capacity of the polymers. Here, we report a layer-by-layer fabrication technique where we separated the active (porous polymer, top) layer and the conductive (carbon, bottom) layer and used these "layered" electrodes in a supercapacitor (SC). Through this approach, direct contact with the electrolyte and polymer material is greatly enhanced. With extensive electrochemical characterisation techniques, we show that the layered electrodes allowed a significant contribution of fast faradic surface reactions to the overall capacitance. The electrochemical performance of the layered-electrode SC outperformed other reported porous polymer-based devices with a specific gravimetric capacitance of $388 \mathrm{~F} \cdot \mathrm{g}^{-1}$ and an outstanding energy density of $65 \mathrm{Wh} \cdot \mathrm{kg}^{-1}$ at a current density of $0.4 \mathrm{~A} \cdot \mathrm{g}^{-1}$. The device also showed outstanding cyclability with $90 \%$ of capacitance retention after 5000 cycles at $1.6 \mathrm{~A} \cdot \mathrm{g}^{-1}$, comparable to the reported porous polymer-based SCs. Thus, the introduction of a layered electrode structure would pave the way for more effective utilisation of porous organic polymers in future energy storage/harvesting and sensing devices by exploiting their nanoporous architecture and limiting the negative effects of the carbon/binder matrix.
\end{abstract}

Keywords: nanoporous polymer; supercapacitor; polyimide; electrochemistry; electrode fabrication; layer-by-layer

\section{Introduction}

The development of low cost, eco-friendly and high-performance energy storage devices is becoming crucial to meeting modern energy storage demand in various applications, including electric vehicles, portable electronics, and power grids. Among them, supercapacitors (SCs) gained significant attention as a promising energy storage device due to their outstanding energy density, power density and long cycle life [1,2]. There are two types of supercapacitor electrodes; one that uses the electric double layer phenomenon and one based on fast faradic surface reactions (pseudocapacitive SCs). High-performance electrode materials should have properties such as an electrical conductivity, a large specific surface area, and good wettability by the electrolyte in both cases. Several materials have been used to fabricate electrodes for supercapacitors, including carbon nanotubes $[1,3]$, graphene [4,5], metal oxides nanoparticles [6-8], transition metal dichalcogenides [9], and carbon-based composites [10,11]. 
Next to these materials are conductive polymers, which have become increasingly popular alternatives as novel active materials for SCs [12-15]. Polymers as active materials for supercapacitors have several advantages over inorganic active materials, such as high theoretical specific capacitance, low cost and ease of large-scale production [16]. Furthermore, small molecular changes in the originating monomeric building blocks can yield a library of polymers with macroscopically different properties, meaning that polymers are highly tuneable. In the field of SCs, this tunability is reflected in the fact that redoxactive segments can be implemented into the polymer-backbone, providing an additional pseudocapacitive energy storage feature. However, conductive polymers still experience limitations, such as poor cycle life and rate kinetics due to repeated swelling and shrinking of the polymer structure during the charge-discharge processes. Additionally, polymer particle aggregation makes it challenging to engineer electrodes that maintain the high theoretical specific capacitance [17].

Alternatively, nanoporous organic polymers emerged as an attractive replacement for linear conductive polymers. Their porous architecture circumvents problems such as low surface areas and aggregation of linear polymers. Thus, nanoporous polymers, in particular, allow the efficient diffusion of ions over an enormous surface area of active material, which significantly improves the capacity $[18,19]$. However, nanoporous polymers introduce the problem of not providing efficient conductive pathways. To alleviate the poor conductivity of these materials, the concept of hybridising them with conductive materials (often carbonbased) has been suggested [20-22]. However, the addition of conductive materials, even when it contributes to the capacitance, reduces the specific capacity of the electrodes below the theoretical value of the nanoporous polymers. Moreover, complex techniques are often required to ensure good adhesion between the conductive agent and redox-active polymer, which hinders their practical applications. Therefore, it is desirable to engineer electrodes that can utilise the high surface area of the porous polymers without sacrificing the specific capacitance.

In the study presented here, we utilised a layer-by-layer electrode fabrication approach to produce bilayer conductive carbon/perylene diimide-based porous polymer electrodes as an alternative to the traditional casting of a pre-mixed paste of the active materials, binder and conductive agent (Figure 1). This electrode preparation approach allows us to prepare electrodes with maximum utilisation of the porous polymer as the active supercapacitive material by enhancing the exposure of active materials to the electrolyte, as has also been exploited by other research groups in the field of 1D polymers [23]. The formation of a layer-by-layer assembly can be explained through intermolecular interactions and is dependent on the chemical nature of the separate layers.

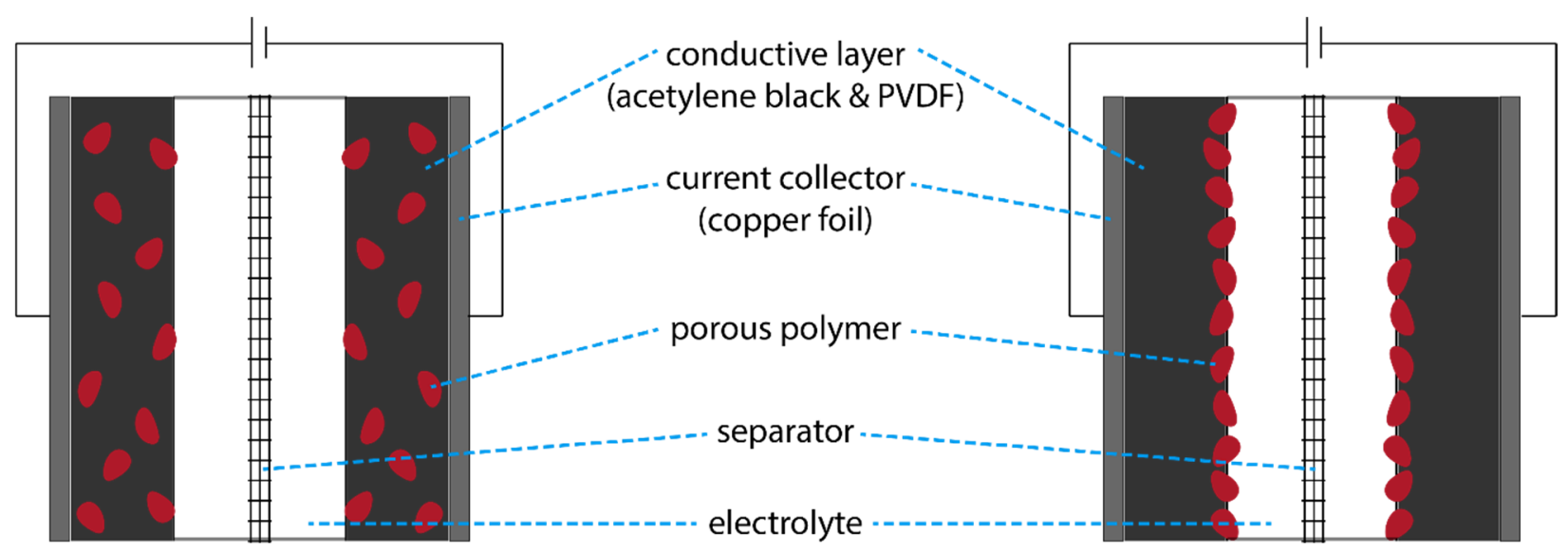

Figure 1. Schematic representation of SCs comprised of two identical electrodes prepared via the conventional mixing method (left) or the layer-by-layer method (right). 
Polyimide porous polymers have been extensively used in gas storage and separation applications [24-27], but the investigation of their potential in the field of energy storage has been limited due to their poor conductivity [28-31]. However, the redox-active segments within their polymer backbone make them interesting to study, especially regarding energy storage applications. One-dimensional perylene diimide-based materials have been widely exploited in the field of photovoltaics and batteries. Often, high electron mobilities in such materials are observed as an effect of their favourable molecular $\pi-\pi$ interactions [32]. In addition, these aromatic polyimides are redox-active and their carbonyl groups can participate in reversible lithium complexation [33]. The combination of both of these properties, integrated within a porous framework, formed the idea that a porous perylene diimide-based polymer may exhibit good electrochemical performance in the application presented here.

\section{Experimental}

\subsection{Materials}

All chemicals were commercially available and used without further treatment. Chemicals including; 3,4,9,10-perylenetetracarboxylic dianhydride (PTCDA); 1,3,5-tris(4-aminoph enyl)benzene ( $\geq 93 \%$, TAPB); isoquinoline; imidazole; meta-cresol and dimethyl carbonate were all purchased from TCI Europe N.V., Zwijndrecht, Belgium. In addition, 1-methyl2-pyrrolidinone (NMP), poly(vinylidene fluoride) (PVDF) and 1.0 M LiPF6 in ethylene carbonate/ethyl methyl carbonate $=1 / 1(v / v)$, battery grade were purchased from Sigma Aldrich, Gillingham, UK.

\subsection{Synthesis of Per-TAPB-PPI}

Scheme 1 shows the chemical structures of TAPB (204 mg, $0.58 \mathrm{mmol})$ and PTCDA (345 mg, $0.88 \mathrm{mmol}$ ), which were weighed and transferred to a $25 \mathrm{~mL}$ ampoule. An amount of $10 \mathrm{~mL}$ of a 1:1 (v/v) mixture of meta-cresol and imidazole was added to the ampoule, and the mixture was sonicated for five minutes. Then, $0.1 \mathrm{~mL}$ of isoquinoline was added, after which the ampoule was degassed via three freeze-pump-thaw cycles. Finally, the ampoule was flame-sealed, allowed to reach room temperature, and placed in an oven at $200{ }^{\circ} \mathrm{C}$ for 5 days. After polymerisation, the precipitate was washed with methanol $(2 \times 30 \mathrm{~mL})$ and acetone $(2 \times 30 \mathrm{~mL})$. The solid was dried in a vacuum oven $\left(60^{\circ} \mathrm{C}\right)$ and subsequently washed in a Soxhlet extractor using THF for $16 \mathrm{~h}$. The resulting polymer, Per-TAPB-PPI, was finally dried overnight in a vacuum oven at $60^{\circ} \mathrm{C}$ and obtained as a dark red powder with a yield of $91 \%$.

\subsection{Molecular and Microstructural Characterisation}

FT-IR spectra were recorded on a PerkinElmer Spectrum 100 FT-IR Spectrometer (Perkin Elmer, Bruxelles, Belgium) with the Universal ATR Accessory over a range of 4000 to $650 \mathrm{~cm}^{-1}$. TGA analyses were performed from room temperature to $860{ }^{\circ} \mathrm{C}$, under nitrogen atmosphere at a heating rate of $10{ }^{\circ} \mathrm{C} / \mathrm{min}$ using a Perkin Elmer TGA 4000 (Perkin Elmer, Bruxelles, Belgium). Before the measurement, the samples were dried at $130{ }^{\circ} \mathrm{C}$ for one hour under a nitrogen atmosphere. The nitrogen sorption isotherms (at 77 K) were measured on a Tristar II 3020 Micromeritics instrument (Micromeritics B.V., Eindhoven, The Netherlands). The porous polymer was degassed before the measurement at $130{ }^{\circ} \mathrm{C}$ under vacuum for $16 \mathrm{~h}$. Quenched-Solid Density Functional Theory (QSDFT) simulations based on the experimental nitrogen adsorption isotherms were performed to calculate the pore size distributions. The chosen model was the carbon, adsorption kernel, slit/cylindrical/spherical pore model, a standardised model in the VersaWin Gas Sorption Data Reduction Software (Quantachrome Instruments, Boynton Beach, FL, USA). Scanning electron microscopy (SEM) images were recorded with a JEOL JSM-840 SEM (JEOL Europe B.V., Nieuw-Vennep, The Netherlands): materials were deposited onto a sticky carbon surface on a flat sample holder, vacuum-degassed, and subjected to gold sputtering. PXRD data were collected on a Rigaku MiniFlex 600 powder diffractometer (Rigaku Innovative 
Technologies, Inc., Auburn Hills, MI, USA) using a Cu-K $\alpha$ source $(\lambda=1.5418 \AA$ ) over the $2 \theta$ range of $5^{\circ}$ to $40^{\circ}$ with a scan rate of $1^{\circ} /$ minute. Contact angle measurements were performed on a Kruss Contact Angle Measuring System G2 (Krüss GmbH, Hamburg, Germany). The solid surfaces used for these measurements were dry-pressed Per-TAPB-PPI pellets (at $300 \mathrm{MPa}$ for $10 \mathrm{~s}$ ), and either water or dimethyl carbonate were used as liquids.<smiles>Nc1ccc(-c2cc(-c3ccc(N)cc3)cc(-c3ccc(N)cc3)c2)cc1</smiles>

TAPB

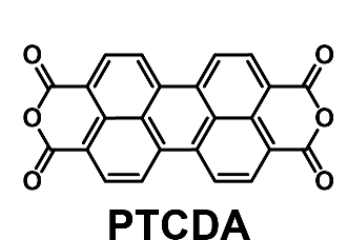

$m$-cresol / imidazole

isoquinoline

PTCDA

$200^{\circ} \mathrm{C}, 5$ days<smiles>Cc1ccc2c3c(ccc(C)c13)C(=O)N(c1ccc(-c3ccc(-c4ccc(-n5c(=O)c6ccc(C)c7c(C)ccc(c(=O)c8ccc(C)c9c(C)ccc(c5=O)c98)c76)cc4)cc3)cc1)C2=O</smiles>

Scheme 1. Formation of porous perylene diimide-based polyimide (Per-TAPB-PPI) through a polycondensation of dianhydride PTCDA with triamine TAPB.

\subsection{Electrochemical Study}

2.4.1. Electrodes Prepared by the Traditional Mixing Method

The active material (Per-TAPB-PPI, $0.5 \mathrm{mg}$ ), acetylene black ( $2 \mathrm{mg}$ ) and PVDF binder were ground together using a small amount of NMP, and the resulting paste was loaded on a copper foil sheet using a doctor blade technique. PVDF act as an effective dispersion agent to connect the electrode species together, ensure the adhesion of materials to the current collectors and provide mechanical integrity for the electrode. The electrodes were dried under vacuum at $50{ }^{\circ} \mathrm{C}$ for around $24 \mathrm{~h}$ and pressed using a mechanical press with a pressure of 75 bar (Metaserv Automatic mounting press, Model C190, Surrey, England).

\subsubsection{Electrodes Prepared by the Layer-by-Layer Method}

Acetylene black $(2 \mathrm{mg})$ and PVDF binder $(0.1 \mathrm{mg})$ were ground using a small amount of NMP, and the resulting paste was loaded on a copper foil sheet using a doctor blade technique. Afterwards, the coated sheets were dried in a vacuum oven at $50{ }^{\circ} \mathrm{C}$ for $2 \mathrm{~h}$ to evaporate the excess NMP solvent. The active material (Per-TAPB-PPI) and PVDF 
binder $(1 \%)$ were sonicated with NMP to form a homogenous slurry and dropwise added to the acetylene black/PVDF layer. The active material loading was $\sim 0.28 \mathrm{mg} \cdot \mathrm{cm}^{-2}$. The electrodes were dried under vacuum at $50{ }^{\circ} \mathrm{C}$ for around $24 \mathrm{~h}$ and pressed using a mechanical press (pressure, 75 bar).

\subsubsection{SC Fabrication}

The symmetrical supercapacitors (SCs) were fabricated by sandwiching two identical working electrodes in a 2032 stainless steel coin cell in both scenarios. The electrolyte was 1 M LiPF6 in ethylene carbonate/ethyl methyl carbonate (EC/EMC) mixture (1:1, $v / v)$ and a glass microfiber filter paper (GF/B, pore size: $1.0 \mu \mathrm{m}$ ) applied as the separator (see Figure 1). All the coin cells were assembled in a dry glove box filled with argon. The supercapacitor performances of as-built coin cells were evaluated on an Iviumstat Electrochemical Interface. Cyclic voltammetry $(\mathrm{CV})$ and galvanostatic charge-discharge (GCD) assessments were applied within the potential range of 0.02-2.22 V and 0.2-1.8 V for the layered SC and traditional mixing SC, respectively. The electrochemical impedance spectroscopy (EIS) analysis was carried out by employing an amplitude of $5 \mathrm{mV}$ over the frequency range from $0.1 \mathrm{~Hz}$ to $400 \mathrm{kHz}$. The cycling stability of the SCs were evaluated over 5000 cycles.

\section{Results and Discussion}

\subsection{Porous Polymer Characterisation}

Imide-bond formation after the polymerisation was confirmed with FT-IR spectroscopy. The characteristic vibrations of the amine-containing monomer TAPB and the anhydride containing monomer PTCDA were not observed in the resulting polymer, while characteristic imide-bond vibrations appeared (see Figure S1, Supporting Information for detailed information). In addition, Per-TAPB-PPI showed excellent stability when exposed to heat, measured by TGA (Figure S2). A temperature of $546{ }^{\circ} \mathrm{C}$ was observed at $5 \%$ weight loss for Per-TAPB-PPI, which is on par with the current state-of-the-art porous polyimides $[24,34]$, and it emphasises the advantage of using imide-linkages in porous polymers. Finally, while the specific use of the monomer TAPB was mainly to promote porous framework formation, it may have added benefits to the final material in terms of optoelectronic and mechanical properties [35].

Nitrogen sorption experiments were conducted to assess the porosity of the synthesised polymer (Figure 2a). The adsorption isotherm showed a steep increase in $\mathrm{N}_{2}$ uptake at the low relative pressure region $\left(<0.05 \mathrm{P} / \mathrm{P}_{0}\right)$, indicating the presence of micropores. Additionally, Per-TAPB-PPI exhibited a further $\left(>0.05 \mathrm{P} / \mathrm{P}_{0}\right)$ slight increase in $\mathrm{N}_{2}$ uptake, which indicates the presence of larger (meso)pores. Pore size distribution (PSD) was calculated by fitting the nitrogen adsorption isotherm to a DFT model (Figure 2b). Specifically, the quenched solid DFT (QSDFT) carbon model was chosen for the PSD calculations since it takes surface roughness into account [36]. Considering that this polymer is largely amorphous (discussed later), the pore geometries are expected to vary, meaning that slit-like, cylindrical, and spherical pores all were considered in the model. Next to the significant presence of micropores within the PSD of Per-TAPB-PPI, a distribution around the maximum population corresponding to a pore size of $2.5 \mathrm{~nm}$ was observed. It should be noted that such PSDs are still estimates based on models that are initially developed for carbon materials. The calculated surface area according to the Brunauer-Emmett-Teller (BET) theory for Per-TAPB-PPI was $411 \mathrm{~m}^{2} \cdot \mathrm{g}^{-1}$ (Figure S3). 

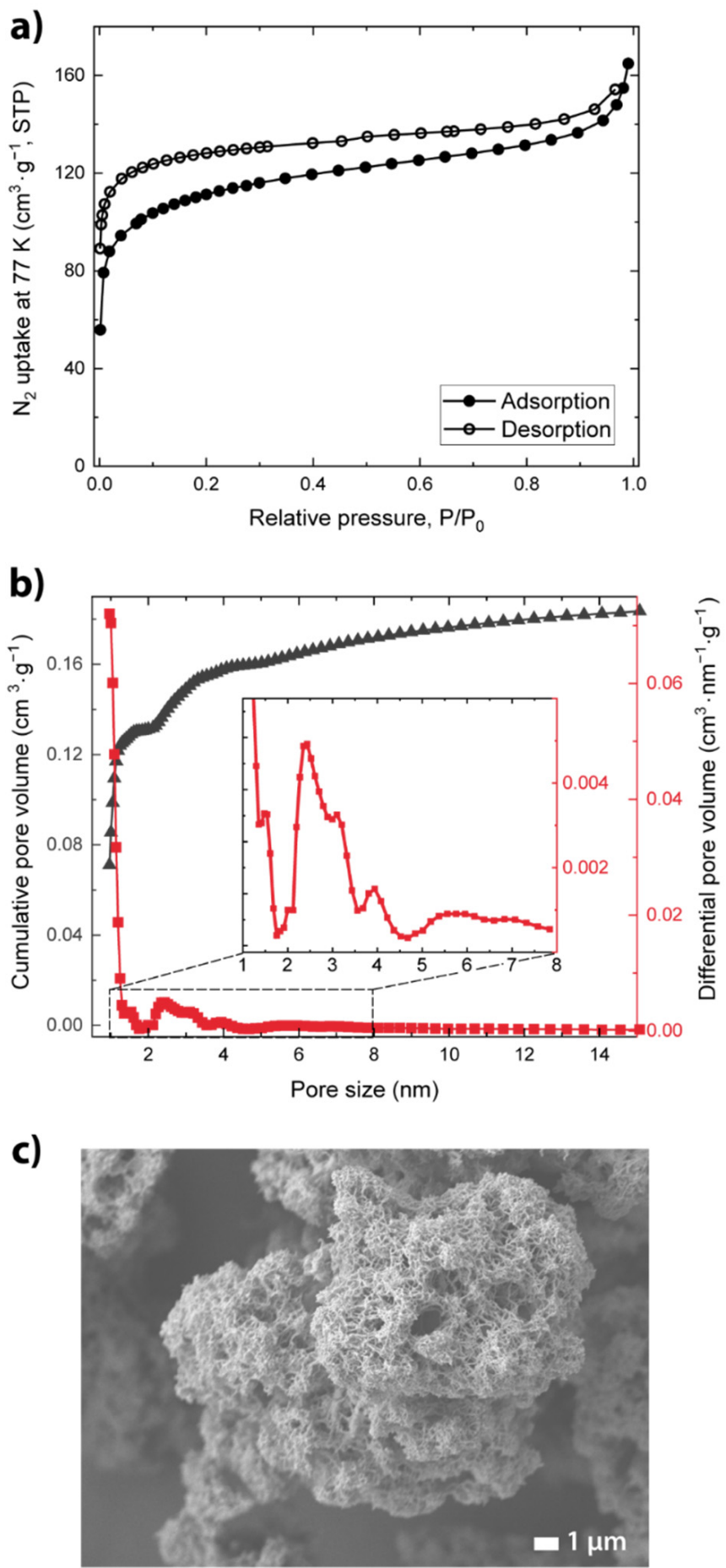

Figure 2. (a) Nitrogen adsorption and desorption isotherm of Per-TAPB-PPI. (b) Pore size distribution (i.e., pore size versus either cumulative pore volume (grey) or differential pore volume (red), including inset with zoom-in) of Per-TAPB-PPI calculated by a QSDFT model with the nitrogen adsorption isotherm input data. (c) SEM micrograph of Per-TAPB-PPI.

While the above results approximate a quantitative description of the nanoporous polymer, we conducted scanning electron microscopy (SEM) measurements to obtain a better qualitative picture of the microscopic porous morphology (Figure 2c). Per-TAPBPPI exhibits large porous aggregate structures originating from interconnected plate-like and spindle-like substructures. In addition to the micro- and mesoporous structures discovered by nitrogen sorption studies described earlier, these SEM images also reveal 
the presence of larger macropores. This hierarchical porous structure (micro-, meso- and macroporosity) promises effective utilisation of the polymer's redox-active sites. The architecture of the polymer was further investigated by the use of powder X-ray diffraction (PXRD, Figure S4). The polymer exhibits a largely amorphous nature with no distinct crystalline domains, while increased diffuse intensity in the lower-angle regions of the PXRD pattern may indicate a short-ranged order of distances similar to the mesopore sizes observed in Figure 2b. Furthermore, the broad signal of Per-TAPB-PPI around $24^{\circ}$ (corresponding to a distance of $3.7 \AA$ ) resembles the interlayer stacking signal often seen in covalent organic frameworks [37], which may indicate a sheet-like character. Although PerTAPB-PPI does not show long-range crystalline order, we do expect that, based on these results and similar patterns observed in the literature, the polymer structure exhibits local stacked regions of neighbouring planes. The porous polymer architecture may influence the wettability of the material, which was investigated next.

The electrolyte wettability of the Per-TAPB-PPI electrode is important for the electrochemical reactions at the electrolyte-electrode interface. We measured this property by performing swelling experiments through a contact angle measurement setup. Dry-pressed polymer pellets were used as solid surfaces. Unfortunately, a rough final surface is inherently linked to this sample preparation, which makes it difficult to obtain a defined contact angle from these measurements. However, the qualitative results that were obtained when comparing water or dimethyl carbonate on a Per-TAPB-PPI surface (Figure S5) exemplified the advantage of using carbonate esters as the electrolyte in the case of electrochemical applications. The referred figure shows the remarkable swelling behaviour of Per-TAPBPPI when exposed to a droplet of dimethyl carbonate. On the other hand, water does not interact with the polymer in such a way and merely wets the polymer as a result of surface roughness. The hydrophobicity (extended aromatic structures) of the material is expected to be the main reason for this, which is strengthened by other research on vapour adsorption experiments of porous polyimides that show poor water uptake (compared to organic solvents such as benzene and hexane, with benzene $/ \mathrm{H}_{2} \mathrm{O}$ selectivity values of 13-28 reported by Li and Wang) [38,39]. Thus, these experiments not only illustrate a good wetting of the polymers with the organic electrolyte but even a pore-opening behaviour and consequently increased access to the polymer's active sites.

\subsection{Supercapacitor Performance}

The extensively characterised porous polymer Per-TAPB-PPI of the previous section has been implemented into two differently prepared electrodes in SCs: via a traditional mixing method and also via the layer-by-layer method. As indicated in the introduction, we expected significant compositional and morphological differences at the electrode's surface that is exposed to the electrolyte. In order to confirm this, SEM micrographs were taken of the surfaces of the two different electrodes (Figure S6a,b). The conventional electrode surface was comparatively smooth and displayed a slightly porous structure that originates from the conductive carbon network. In contrast, the layered electrode revealed the presence of embedded aggregated porous polymer particles over the surface. To verify that this assumption, energy dispersive X-ray spectroscopy (EDX) was used to assess both different electrode surfaces (Figure S6c,d). The layer-by-layer-prepared electrodes contained a significantly higher amount of oxygen atoms at the surface, originating from the carbonyl groups of Per-TAPB-PPI. The larger availability of these redox-active carbonyl-groups is expected to improve the electrochemical performance of the layer-by-layer-prepared SCs when compared to the SC comprised of traditionally prepared electrodes.

The electrochemical performances of the SCs were studied using LiPF6 salt dissolved in a mixture of EC/EMC solvents as the electrolyte. Figure 3a,b show the CV curves for the Per-TAPB-PPI-based SCs prepared by the traditional mixing method and the layerby-layer method, respectively, both at scan rates ranging from 10 to $200 \mathrm{mV} \cdot \mathrm{s}^{-1}$. The $\mathrm{CV}$ curves of the SC fabricated from the traditional method show an electrical double layer capacitance (EDLC) with a minute pseudocapacitive contribution, which is apparent 
from the absence of clear redox peaks. In contrast, the layer-by-layer prepared Per-TAPBPPI SC (Figure 3b) delivered EDLC, as well as a clear pseudocapacitance along with distinctive reversible redox humps at $\sim 0.95 \mathrm{~V}$ for cathodic and $\sim 1.20$ and $\sim 1.60 \mathrm{~V}$ for anodic peaks. First, the EDLC of both SCs can be attributed to the large surface area and nanoporous architecture of Per-TAPB-PPI, allowing the diffusion and adsorption of a high number of $\mathrm{Li}^{+}$ions. In addition, the contribution of acetylene black could be recognised in both scenarios in terms of EDLC, with a more prominent involvement expected in the traditional SC. Additionally, the presence of a sizeable $\pi$-conjugated system could further facilitate the electrical conductivity and hence increase the $\mathrm{Li}^{+}$ion adsorption to the active material $[40,41]$. Secondly, the pronounced pseudocapacitive contribution in the layered SC $\mathrm{CVs}$ indicates surface faradic redox reactions of $\mathrm{Li}^{+}$ions with carbonyl functionalities of the perylene diimide-based polymers (Scheme 2). This also explains the overall higher current compared to the traditional Per-TAPB-PPI SC. Furthermore, the minor faradic contribution in the conventional SC is likely caused by the hindrance of the acetylene black/PVDF matrix, limiting the availability of the active sites of the Per-TAPB-PPI polymer.

To further investigate the electrochemical performance of the Per-TAPB-PPI SCs, GCD measurements were carried out at various current densities (Figure 3c,d). For the traditional SC, a nearly perfect triangular-shaped profile was observed. Such a curved shape indicates a pure EDLC character, which reinforces the CV results. On the other hand, the GCD curves of the layered electrode displayed prominent nonlinear characteristics, confirming the pseudocapacitive contribution. Moreover, the layer-by-layer prepared SC exhibited longer discharge times at comparatively higher current densities than those of the traditional SC. Additionally, the layered SC's discharge curves show a rapid voltage decay at the early stage due to non-faradic electrostatic discharge (i.e., adsorption and diffusion processes) and a final slow potential drop due to the faradic discharge [42,43]. From these GCD plots, the specific capacitance $C_{S}$ of both SCs was estimated using Equation (1) [1].

$$
\mathrm{Cs}=4 \frac{I}{m(d V / d t)}
$$

where $I(\mathrm{~A})$ is the constant discharge current, $t(\mathrm{~s})$ is the discharge time, $V(\mathrm{~V})$ is the potential window, and $m(\mathrm{~g})$ is the active material mass in both electrodes. Furthermore, the energy (E) and the power $(\mathrm{P})$ densities for the $\mathrm{SC}$ cells were estimated using $\mathrm{E}=0.125 \mathrm{C}_{\mathrm{S}}(\Delta \mathrm{V})^{2} / 3.6$ and $\mathrm{P}=3600 \mathrm{E} / \Delta \mathrm{t}$, respectively $[44,45]$.

As presented in Figure $3 e$, the highest specific capacitance $C_{s}$ of the traditional PerTAPB-PPI-based SC was $178 \mathrm{~F} \cdot \mathrm{g}^{-1}$ at a current density of $0.1 \mathrm{~A} \cdot \mathrm{g}^{-1}$ while the layered SC exhibited an outstanding capacitance of $388 \mathrm{~F} \mathrm{~g}^{-1}$ at a specific current density of $0.4 \mathrm{~A} \cdot \mathrm{g}^{-1}$. The resulting higher $C_{S}$ of the layered $S C$ follows directly from the observations in the $\mathrm{CV}$ and GCD plots, with the most prominent reason being the additional pseudocapacitive behaviour that this $\mathrm{SC}$ shows. The significant differences in $\mathrm{C}_{\mathrm{S}}$ values between the differently prepared electrodes exemplify the electrode fabrication method greatly influences its electrochemical performance. In our case, Per-TAPB-PPI benefits from being in direct contact with the electrolyte (rather than being obstructed in the acetylene black/PVDF matrix), since the fast faradic surface reactions are then better exploited. Moreover, the energy (E) and power (P) densities for the SC devices were evaluated and plotted in Figure 3f. As presented, the layer-by-layer prepared Per-TAPB-PPI SC device exhibited an excellent maximum energy density of $65 \mathrm{Wh} \cdot \mathrm{kg}^{-1}$ at $0.4 \mathrm{~A} \cdot \mathrm{g}^{-1}$ and a high power density of $2.2 \mathrm{~kW} \cdot \mathrm{kg}^{-1}$ at $1.6 \mathrm{~A} \cdot \mathrm{g}^{-1}$. Similarly, the maximum energy density for the traditionally prepared Per-TAPB-PPI- SC device was only $16 \mathrm{Wh} \cdot \mathrm{kg}^{-1}$ at a current density of $0.1 \mathrm{~A} \cdot \mathrm{g}^{-1}$ along with a power density of $2.6 \mathrm{~kW} \cdot \mathrm{kg}^{-1}$ at $1.6 \mathrm{~A} \cdot \mathrm{g}^{-1}$. According to the results, we demonstrated a substantial energy density improvement in the layer-by-layer prepared Per-TAPB-PPI SC device, confirming the enhanced electrochemical performance. 

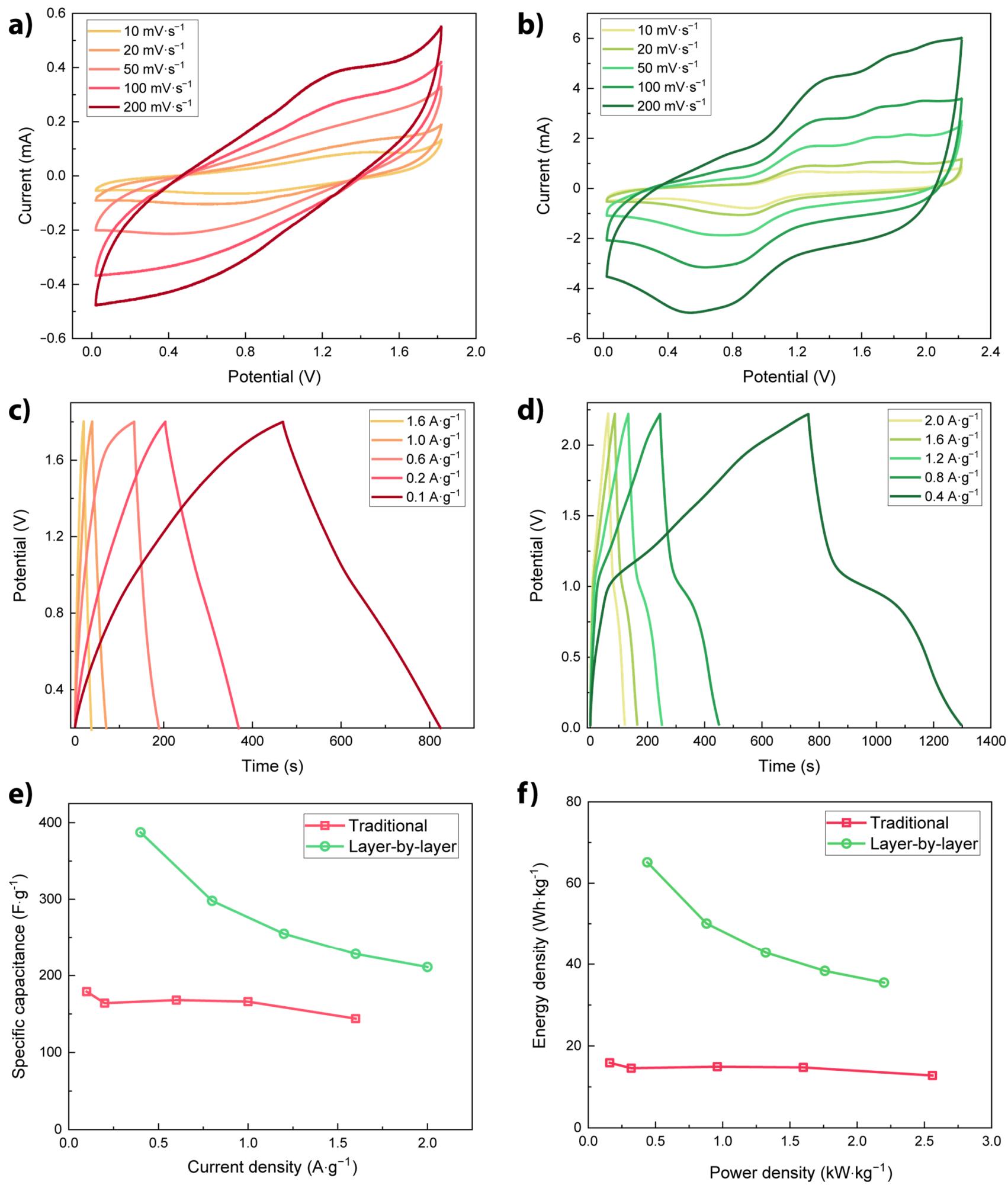

Figure 3. Cyclic voltammograms (scan rates 10 to $200 \mathrm{mV} \mathrm{s}^{-1}$ ) of SCs composed of Per-TAPBPPI-based electrodes prepared via (a) a traditional mixing method or (b) a layer-by-layer method. Galvanostatic charge-discharge profiles at different current densities of Per-TAPB-PPI-based electrodes prepared via (c) a traditional mixing method or (d) a layer-by-layer method. (e) Corresponding specific capacitance at different current densities. (f) Energy and power densities of SC devices. 


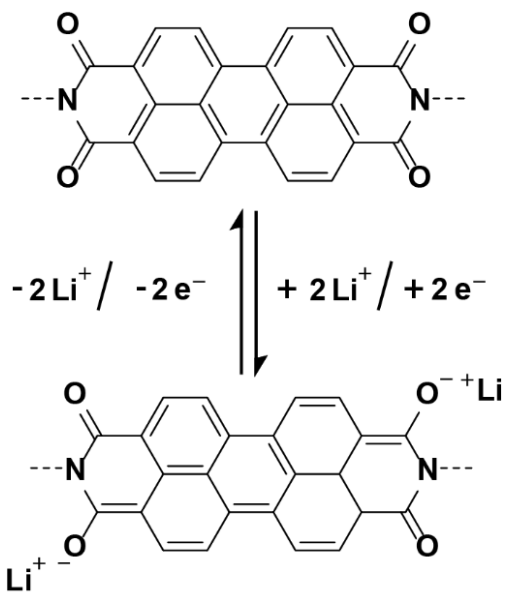

Scheme 2. Faradic reaction mechanism of perylene diimide-based porous polymers with Li-ions.

The cyclability test of the electrode prepared by the traditional and by the layer-bylayer methods are illustrated in Figure 4. Mass loadings of the electrodes were optimised for a balanced capacitance and cycle stability. During the cyclic test, the layered SC was first run at a current density of $0.8 \mathrm{~A} \cdot \mathrm{g}^{-1}$ over 5 cycles to activate the $\mathrm{SC}$ and a remarkable $90.6 \%$ capacitance retention was observed after 5000 cycles at a current density of $1.6 \mathrm{~A} \cdot \mathrm{g}^{-1}$. Similarly, the traditional SC was activated at $0.4 \mathrm{~A} \cdot \mathrm{g}^{-1}$ over 5 cycles, and the $93.9 \%$ capacitance retention was obtained after 5000 cycles at a current density of $0.8 \mathrm{~A} \cdot \mathrm{g}^{-1}$. The layered SC demonstrates the excellent electrochemical performance during cycling at a higher current density compared to the traditional electrode, which displays fast electrochemical kinetics that arise from the direct contact between the electrolyte and the porous polymer layer. The calculated cyclability of Per-TAPB-PPI are comparable with other COF-based electrodes prepared without any hybridisation with carbon materials as illustrated in Table S1 (supporting information), indicating the feasibility of our new materials and electrode fabrication method on improving the capacitance without sacrificing the cyclability of the electrode. A detailed post-mortem analysis of the electrode surface after cycling experiments, as has been studied by the group of Yoo [46], is able to provide additional insights in the effect of induced stress and strain to porous polymer materials. Our group is currently investigating this and aims to report this in future work.

A final characterisation method we used to elucidate the electrochemical performance of the differently prepared electrodes was electrochemical impedance spectroscopy (EIS), since it provides information on the equilibrated states of the SCs. The experiments were performed over a frequency range of $0.01-400 \mathrm{kHz}$, and the resulting impedance plots (Nyquist plots) are presented in Figure 5a,b. Overall, the near-vertical lines for the lower frequency range (Figure 5a) of both SCs indicate capacitive behaviour and efficient ion diffusion between the electrolyte and the electrode surface [47-51]. Furthermore, the higher frequencies in the Nyquist plots (Figure $5 b$ ) provide information about the serial $\left(R_{S}\right)$ and charge transfer $\left(\mathrm{R}_{\mathrm{CT}}\right)$ resistances in the electrodes. Here, the intersect with the $\mathrm{Z}^{\prime}$ axis gives $R_{S}$, which originates from the internal resistance of the electrode materials. Both electrodes show relatively low internal resistances: $4.6 \Omega$ for the layered and $6.2 \Omega$ for the traditional electrode. These results suggest the presence of efficient conductive pathways between the polymer ( $\pi$ electrons) and conductive carbon. In addition, a slightly higher $\mathrm{R}_{\mathrm{CT}}$ was observed for the layered electrode $(3.0 \Omega$ ) compared to that of the traditional electrode $(0.6 \Omega)$. The lower charge transfer resistance in the traditional electrode is likely an effect of the large contribution by the highly conductive carbon matrix. Furthermore, the $\mathrm{R}_{\mathrm{CT}}$ of both the layered and traditional Per-TAPB-PPI SCs were significantly lower than that of previously reported organic polymer electrodes including a hydroquinonebased COF TpPa- $(\mathrm{OH})(37.5 \Omega)$ [52], and a benzimidazole-based COF TpDAB (24.8 $\Omega$ ) [53]. This contrast is likely an effect of molecular structure, rather than polymer architecture 
or device preparation procedure, since perylene diimide derivatives are among the most common acceptor units because of their high electron affinity and electron mobility. Finally, additional experiments on a range of related polymers with perylene diimide units are required to link the promising performance of this polymer to its structure.

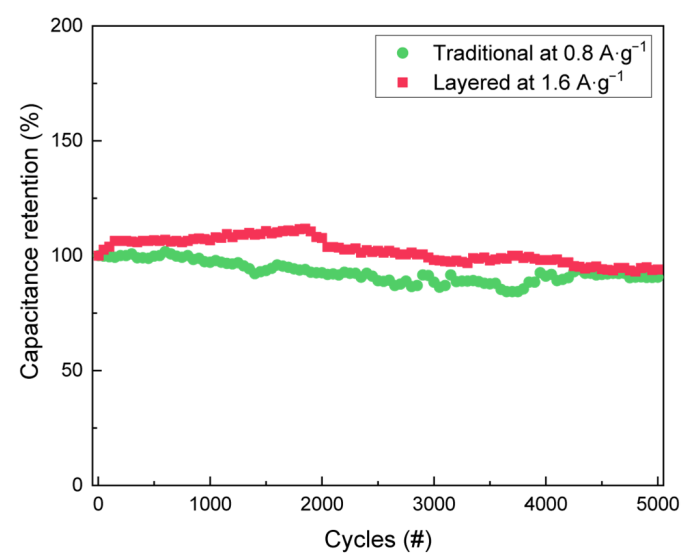

Figure 4. Cyclic stability of layered and traditional Per-TAPB-PPI supercapacitors.
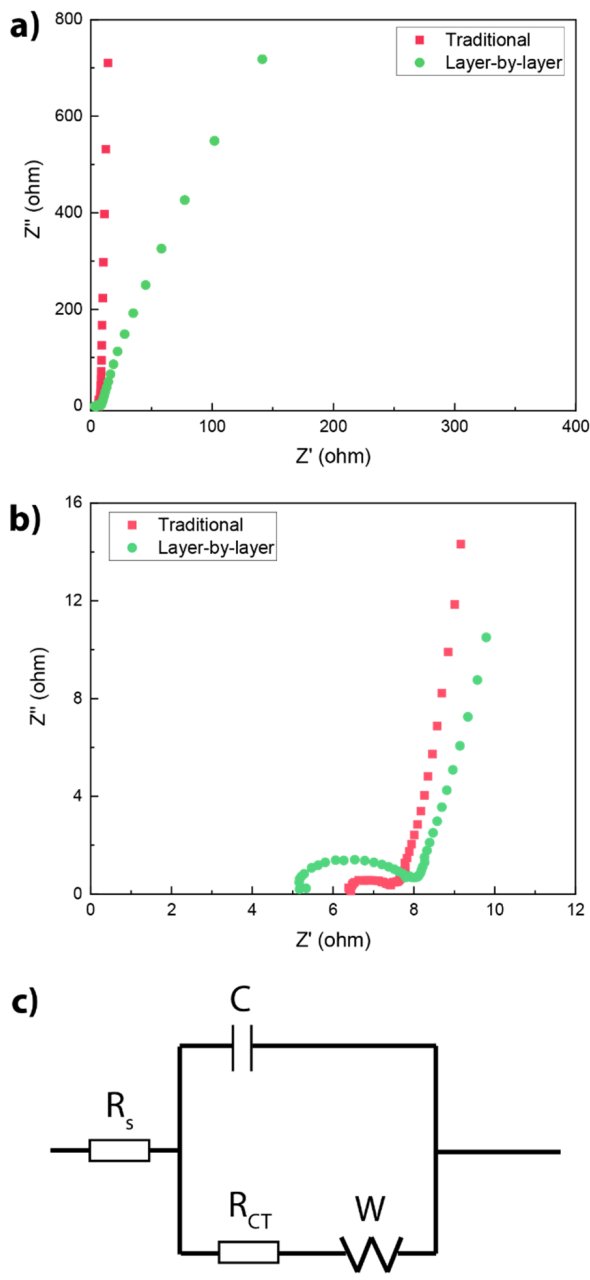

Figure 5. Electrochemical impedance spectra of SCs composed of Per-TAPB-PPI-based electrodes prepared via a conventional mixing or a layer-by-layer method. (a) Focused on the lower frequency measurements. (b) Focused on higher frequency measurements. (c) The equivalent circuit modelling of the EIS spectra. 


\section{Conclusions}

In this research, we investigated the effects of a novel layer-by-layer electrode preparation method on the performance of a porous polymer-based supercapacitor. By physically separating the redox-active porous polymer layer and the electron-conducting carbon layer, we created better accessible pathways for ions to diffuse towards the electrodes. As a result, faradic surface reactions were contributing significantly to the overall supercapacitor performance. In addition, by exposing the polymer directly to the electrolyte, the advantages of a porous polyimide polymer (i.e., nanoporosity and redox-activity) were much more exploited. In planned future research, we aim to use a variety of porous polymers in layerby-layer prepared electrodes to elucidate the correlation between polymer architecture and electrochemical performance. We expect that this electrode preparation method allows the observation of more apparent effects originating from the polymer itself, which bridges the gap between molecular structure and electrochemical performance.

Supplementary Materials: The following supporting information can be downloaded at: https: / / www.mdpi.com/article/10.3390/ma15010004/s1. Figure S1: FT-IR spectra of Per-TAPB-PPI and its originating monomers PTCDA and TAPB; Figure S2: TGA profile of Per-TAPB-PPI heated from 30 to $860{ }^{\circ} \mathrm{C}$ at a rate of $10^{\circ} \mathrm{C} / \mathrm{min}$ under constant flow of nitrogen gas; Figure S3: BET plot of PerTAPB-PPI; Figure S4: PXRD pattern of Per-TAPB-PPI; Figure S5: (a) Contact angle measurements of dry-pressed Per-TAPB-PPI pellets with either dimethyl carbonate or water as liquid. Images were taken after 1,8 , and $15 \mathrm{~s}$. (b) Zoom-in of the $1 \mathrm{~s}$ and $15 \mathrm{~s}$ photographs regarding the water experiment showing droplet spreading. (c) Zoom-in of the $1 \mathrm{~s}$ and $15 \mathrm{~s}$ photographs regarding the dimethyl carbonate experiment showing swelling of the pellet; Figure S6: (a) SEM micrograph of the surface of a Per-TAPB-PPI-based electrode prepared by a traditional mixing method. (b) SEM micrograph of the surface of a Per-TAPB-PPI-based electrode prepared by a layer-by-layer method. (c) SEM micrograph of the surface of a Per-TAPB-PPI-based electrode prepared by a traditional mixing method, including EDX mapping filtering for carbon and oxygen. (d) SEM micrograph of the surface of a Per-TAPB-PPI-based electrode prepared by a layer-by-layer method, including EDX mapping filtering for carbon and oxygen; Table S1: Comparison of our supercapacitor systems with previously reported COF based electrodes.

Author Contributions: Conceptualisation, A.N., S.v.d.Z. and A.A.; methodology, A.N. and A.A.; validation, A.N., S.v.d.Z. and A.A.; formal analysis, N.F. and H.V.; investigation, N.F., H.V. and A.A.; resources, A.N., S.v.d.Z. and A.A.; data curation, N.F. and H.V.; writing-original draft preparation, N.F., H.V. and A.A.; writing-review and editing, A.N., S.v.d.Z. and A.A.; visualisation, N.F. and H.V.; supervision, A.N., S.v.d.Z. and A.A.; project administration, S.v.d.Z. and A.A. All authors have read and agreed to the published version of the manuscript.

Funding: This research received no external funding.

Institutional Review Board Statement: Not applicable.

Informed Consent Statement: Not applicable.

Data Availability Statement: Data is contained within the article.

Conflicts of Interest: The authors declare no conflict of interest.

\section{References}

1. Deng, L.; Young, R.J.; Kinloch, I.A.; Abdelkader, A.M.; Holmes, S.M.; De Haro-Del Rio, D.A.; Eichhorn, S.J. Supercapacitance from Cellulose and Carbon Nanotube Nanocomposite Fiber. ACS Appl. Mater. Interfaces 2013, 5, 9983-9990. [CrossRef]

2. Zhang, C.; Qiao, Y.; Xiong, P.; Ma, W.; Bai, P.; Wang, X.; Li, Q.; Zhao, J.; Xu, Y.; Chen, Y.; et al. Conjugated Microporous Polymers with Tunable Electronic Structure for High-Performance Potassium-Ion Batteries. ACS Nano 2019, 13, 745-754. [CrossRef] [PubMed]

3. Aval, L.F.; Ghoranneviss, M.; Pour, G.B. High-performance supercapacitors based on the carbon nanotubes, graphene and graphite nanoparticles electrodes. Heliyon 2018, 4, e00862. [CrossRef] [PubMed]

4. Zhang, L.L.; Zhou, R.; Zhao, X.S. Graphene-based materials as supercapacitor electrodes. J. Mater. Chem. 2010, $20,5983-5992$. [CrossRef] 
5. Abdelkader, A.M. Electrochemical synthesis of highly corrugated graphene sheets for high performance supercapacitors. J. Mater. Chem. A 2015, 3, 8519-8525. [CrossRef]

6. Zhou, Y.; Lee, I.; Kim, D.; Han, S.; Kim, J.K.; Lee, D.; Ko, S.W.; Pyo, S.G.; Son, H.; Yoon, S. Direct Synthesis of Carbon Sheathed Tungsten Oxide Nanoparticles via Self-Assembly Route for High Performance Electrochemical Charge Storage Electrode. J. Nanosci. Nanotechnol. 2017, 17, 389-397. [CrossRef]

7. Sharma, V.; Singh, I.; Chandra, A. Hollow nanostructures of metal oxides as next generation electrode materials for supercapacitors. Sci. Rep. 2018, 8, 1307. [CrossRef]

8. Daneshvar, F.; Aziz, A.; Abdelkader, A.M.; Zhang, T.; Sue, H.J.; Welland, M.E. Porous $\mathrm{SnO}_{2}-\mathrm{Cu}_{\mathrm{x}} \mathrm{O}$ nanocomposite thin film on carbon nanotubes as electrodes for high performance supercapacitors. Nanotechnology 2019, 30, 015401. [CrossRef]

9. Lin, L.W.; Lei, S.; Zhang, Y.; Liu, G.G.; Wallace, J.C. Two-dimensional transition metal dichalcogenides in supercapacitors and secondary batteries. Energy Storage Mater. 2019, 19, 408-423. [CrossRef]

10. Zheng, J.; Yang, T.; Chen, J.; Xu, J.; Jin, T. Constructing $\mathrm{Fe}_{3} \mathrm{O}_{4} /$ Nitrogen-doped Graphene Composites with Rich Pyrrolic Nitrogen for Excellent Supercapacitor Performance. Int. J. Electrochem. Sci. 2020, 15, 6207-6216. [CrossRef]

11. Sun, G.; Liu, J.; Zhang, X.; Wang, X.; Li, H.; Yu, Y.; Huang, W.; Zhang, H.; Chen, P. Fabrication of Ultralong Hybrid Microfibers from Nanosheets of Reduced Graphene Oxide and Transition-Metal Dichalcogenides and their Application as Supercapacitors. Angew. Chem. Int. Ed. 2014, 53, 12576-12580.

12. Biswas, S.; Drzal, L.T. Multilayered Nanoarchitecture of Graphene Nanosheets and Polypyrrole Nanowires for High Performance Supercapacitor Electrodes. Chem. Mater. 2010, 22, 5667-5671. [CrossRef]

13. Wang, K.; Huang, J.; Wei, Z. Conducting Polyaniline Nanowire Arrays for High Performance Supercapacitors. J. Phys. Chem. C 2010, 114, 8062-8067. [CrossRef]

14. Sajedi-Moghaddam, A.; Saievar-Iranizad, E.; Pumera, M. Two-dimensional transition metal dichalcogenide/conducting polymer composites: Synthesis and applications. Nanoscale 2017, 9, 8052-8065. [CrossRef]

15. Qi, K.; Hou, R.; Zaman, S.; Qiu, Y.; Xia, B.Y.; Duan, H. Construction of Metal-Organic Framework/Conductive Polymer Hybrid for All-Solid-State Fabric Supercapacitor. ACS Appl. Mater. Interfaces 2018, 10, 18021-18028. [CrossRef]

16. Meng, Q.; Cai, K.; Chen, Y.; Chen, L. Research progress on conducting polymer based supercapacitor electrode materials. Nano Energy 2017, 36, 268-285. [CrossRef]

17. Snook, G.A.; Kao, P.; Best, A.S. Conducting-polymer-based supercapacitor devices and electrodes. J. Power Sources 2011, 196, 1-12. [CrossRef]

18. Buyukcakir, O.; Je, S.H.; Choi, D.S.; Talapaneni, S.N.; Seo, Y.; Jung, Y.; Polychronopoulou, K.; Coskun, A. Porous cationic polymers: The impact of counteranions and charges on $\mathrm{CO}_{2}$ capture and conversion. Chem. Commun. 2016, 52, 934-937. [CrossRef] [PubMed]

19. Jagadesan, P.; Eder, G.; McGrier, P.L. The excited-state intramolecular proton transfer properties of three imine-linked twodimensional porous organic polymers. J. Mater. Chem. C 2017, 5, 5676-5679. [CrossRef]

20. Sun, B.; Liu, J.; Cao, A.; Song, W.; Wang, D. Interfacial synthesis of ordered and stable covalent organic frameworks on aminofunctionalized carbon nanotubes with enhanced electrochemical performance. Chem. Commun. 2017, 53, 6303-6306. [CrossRef] [PubMed]

21. Jin, J.; Mu, H.; Wang, W.; Li, X.; Cheng, Q.; Wang, G. Long-life flexible supercapacitors based on nitrogen-doped porous graphene@ $\pi$-conjugated polymer film electrodes and porous quasi-solid-state polymer electrolyte. Electrochim. Acta 2019, 317, 250-260. [CrossRef]

22. Tahir, M.; He, L.; Haider, W.A.; Yang, W.; Hong, X.; Guo, Y.; Pan, X.; Tang, H.; Li, Y.; Mai, L. Co-Electrodeposited porous PEDOT-CNT microelectrodes for integrated microsupercapacitors with high energy density, high rate capability, and long cycling life. Nanoscale 2019, 11, 7761-7770. [CrossRef]

23. Kulandaivalu, S.; Suhaimi, N.; Sulaiman, Y. Unveiling high specifc energy supercapacitor from layer-by-layer assembled polypyrrole/graphene oxide I polypyrrole/manganese oxide electrode material. Sci. Rep. 2019, 9, 4884. [CrossRef]

24. Rao, K.V.; Haldar, R.; Kulkarni, C.; Maji, T.K.; George, S.J. Perylene Based Porous Polyimides: Tunable, High Surface Area with Tetrahedral and Pyramidal Monomers. Chem. Mater. 2012, 24, 969-971. [CrossRef]

25. Jiang, L.; Tian, Y.; Sun, T.; Zhu, Y.; Ren, H.; Zou, X.; Ma, Y.; Meihaus, K.R.; Long, J.R.; Zhu, G. A Crystalline Polyimide Porous Organic Framework for Selective Adsorption of Acetylene over Ethylene. J. Am. Chem. Soc. 2018, 140, 15724-15730. [CrossRef] [PubMed]

26. Liebl, M.R.; Senker, J. Microporous Functionalized Triazine-Based Polyimides with High $\mathrm{CO}_{2}$ Capture Capacity. Chem. Mater. 2013, 25, 970-980. [CrossRef]

27. Luo, Y.; Li, B.; Liang, L.; Tan, B. Synthesis of cost-effective porous polyimides and their gas storage properties. Chem. Commun. 2011, 47, 7704-7706. [CrossRef]

28. Tian, D.; Zhang, H.Z.; Zhang, D.S.; Chang, Z.; Han, J.; Gao, X.P.; Bu, X.H. Li-ion storage and gas adsorption properties of porous polyimides (PIs). RSC Adv. 2014, 4, 7506-7510. [CrossRef]

29. Xu, F.; Jin, S.; Zhong, H.; Wu, D.; Yang, X.; Chen, X.; Wei, H.; Fu, R.; Jiang, D. In-situ epitaxial growth of graphene/h-BN van der Waals heterostructures by molecular beam epitaxy. Sci. Rep. 2015, 5, 14760.

30. Li, Z.; Zhou, J.; Xu, R.; Liu, S.; Wang, Y.; Li, P.; Wu, W.; Wu, M. Synthesis of three dimensional extended conjugated polyimide and application as sodium-ion battery anode. Chem. Eng. J. 2016, 287, 516-522. [CrossRef] 
31. Van der Jagt, R.; Vasileiadis, A.; Veldhuizen, H.; Shao, P.; Feng, X.; Ganapathy, S.; Habisreutinger, N.C.; van der Veen, M.A.; Wang, C.; Wagemaker, M.; et al. Synthesis and Structure-Property Relationships of Polyimide Covalent Organic Frameworks for Carbon Dioxide Capture and (Aqueous) Sodium-Ion Batteries. Chem. Mater. 2021, 33, 818-833. [CrossRef]

32. Venkateswararao, A.; Liu, S.W.; Wong, K.T. Organic polymeric and small molecular electron acceptors for organic solar cells. Mater. Sci. Eng. R Rep. 2018, 124, 1-57.

33. Iordache, A.; Delhorbe, V.; Bardet, M.; Dubois, L.; Gutel, T.; Picard, L. Perylene-Based All-Organic Redox Battery with Excellent Cycling Stability. ACS Appl. Mater. Interfaces 2016, 8, 22762-22767. [CrossRef]

34. Fang, Q.; Wang, J.; Gu, S.; Kaspar, R.B.; Zhuang, Z.; Zheng, J.; Guo, H.; Qiu, S.; Yan, Y. 3D Porous Crystalline Polyimide Covalent Organic Frameworks for Drug Delivery. J. Am. Chem. Soc. 2015, 137, 8352-8355. [CrossRef] [PubMed]

35. Zhao, W.; Cao, X.; Huang, J.; Wen, J.; He, Y.; Zha, J.; Li, R.K.Y.; Wu, W. Construction of micro-branched crosslink fluorinated polyimide with ultra-low dielectric permittivity and enhanced mechanical properties. Express Polym. Lett. 2022, 16, 142-151. [CrossRef]

36. Neimark, A.V.; Lin, Y.; Ravikovitch, P.I.; Thommes, M. Quenched solid density functional theory and pore size analysis of micro-mesoporous carbons. Carbon N. Y. 2009, 47, 1617-1628. [CrossRef]

37. Spitler, E.L.; Koo, B.T.; Novotney, J.L.; Colson, J.W.; Uribe-Romo, F.J.; Gutierrez, G.D.; Clancy, P.; Dichtel, W.R. A 2D Covalent Organic Framework with 4.7-nm Pores and Insight into Its Interlayer Stacking. J. Am. Chem. Soc. 2011, 133, 19416-19421. [CrossRef]

38. Li, G.; Wang, Z. Microporous Polyimides with Uniform Pores for Adsorption and Separation of $\mathrm{CO}_{2}$ Gas and Organic Vapors. Macromolecules 2013, 46, 3058-3066. [CrossRef]

39. Shen, C.; Bao, Y.; Wang, Z. Tetraphenyladamantane-based microporous polyimide for adsorption of carbon dioxide, hydrogen, organic and water vapors. Chem. Commun. 2013, 49, 3321-3323. [CrossRef]

40. Bhanja, P.; Das, S.K.; Bhunia, K.; Pradhan, D.; Hayashi, T.; Hijikata, Y.; Irle, S.; Bhaumik, A. A New Porous Polymer for Highly Efficient Capacitive Energy Storage. ACS Sustain. Chem. Eng. 2018, 6, 202-209. [CrossRef]

41. Vadiyar, M.M.; Liu, X.; Ye, Z. Macromolecular Polyethynylbenzonitrile Precursor-Based Porous Covalent Triazine Frameworks for Superior High-Rate High-Energy Supercapacitors. ACS Appl. Mater. Interfaces 2019, 11, 45805-45817. [CrossRef] [PubMed]

42. Wu, Y.; Yan, D.; Zhang, Z.; Matsushita, M.M.; Awaga, K. Electron Highways into Nanochannels of Covalent Organic Frameworks for High Electrical Conductivity and Energy Storage. ACS Appl. Mater. Interfaces 2019, 11, 7661-7665. [CrossRef]

43. Chang, I.C.; Chen, T.T.; Yang, M.H.; Chiu, H.T.; Lee, C.Y. Self-powered electrochemical deposition of Cu@Ni(OH $)_{2}$ nanobelts for high performance pseudocapacitors. J. Mater. Chem. A 2014, 2, 10370-10374. [CrossRef]

44. Fernando, N.; Chinnappan, A.; Aziz, A.; Abdelkader, A.; Ramakrishna, S.; Welland, M.E. Flexible free-standing Ni-Mn oxide antenna decorated CNT/nanofiber membrane for high-volumetric capacitance supercapacitors. Nanoscale 2021, 13, 19038-19048 [CrossRef]

45. Wang, H.; Yi, H.; Chen, X.; Wang, X. Asymmetric supercapacitors based on nano-architectured nickel oxide/graphene foam and hierarchical porous nitrogen-doped carbon nanotubes with ultrahigh-rate performance. J. Mater. Chem. A 2014, 2, 3223-3230. [CrossRef]

46. Ramakrishnan, S.; Velusamy, D.B.; Sengodan, S.; Nagaraju, G.; Kim, D.H.; Kim, A.R.; Yoo, D.J. Rational design of multifunctional electrocatalyst: An approach towards efficient overall water splitting and rechargeable flexible solid-state zinc-air battery. Appl. Catal. B Environ. 2022, 300, 120752. [CrossRef]

47. Basiricò, L.; Lanzara, G. Moving towards high-power, high-frequency and low-resistance CNT supercapacitors by tuning the CNT length, axial deformation and contact resistance. Nanotechnology 2012, 23, 305401. [CrossRef]

48. Gao, Y.; Zhi, C.; Cui, P.; Zhang, K.A.I.; Lv, L.P.; Wang, Y. Halogen-functionalized triazine-based organic frameworks towards high performance supercapacitors. Chem. Eng. J. 2020, 400, 125967. [CrossRef]

49. Li, K.B.; Shi, D.W.; Cai, Z.Y.; Zhang, G.L.; Huang, Q.A.; Liu, D.; Yang, C.P. Studies on the equivalent serial resistance of carbon supercapacitor. Electrochim. Acta 2015, 174, 596-600. [CrossRef]

50. Taberna, P.L.; Simon, P.; Fauvarque, J.F. Electrochemical Characteristics and Impedance Spectroscopy Studies of Carbon-Carbon Supercapacitors. J. Electrochem. Soc. 2003, 150, A292. [CrossRef]

51. Gao, Y.; Li, Y.; An, H.; Feng, Y.; Feng, W. Copolymers of aniline and 2-aminoterephthalic acid as a novel cathode material for hybrid supercapacitors. RSC Adv. 2017, 7, 8762-8770. [CrossRef]

52. Chandra, S.; Roy Chowdhury, D.; Addicoat, M.; Heine, T.; Paul, A.; Banerjee, R. Molecular Level Control of the Capacitance of Two-Dimensional Covalent Organic Frameworks: Role of Hydrogen Bonding in Energy Storage Materials. Chem. Mater. 2017, 29, 2074-2080. [CrossRef]

53. Patra, B.C.; Khilari, S.; Satyanarayana, L.; Pradhan, D.; Bhaumik, A. A new benzimidazole based covalent organic polymer having high energy storage capacity. Chem. Commun. 2016, 52, 7592-7595. [CrossRef] [PubMed] 